University of South Carolina

Scholar Commons

1997

\title{
Semiclassical Approach to the Hydrogen-exchange Reaction- Reactive and Transition-state Dynamics
}

\author{
Sophya V. Garashchuk \\ University of South Carolina - Columbia, garashch@mailbox.sc.edu \\ Grossmann Frank \\ David Tannor
}

Follow this and additional works at: https://scholarcommons.sc.edu/chem_facpub

Part of the Chemistry Commons

Publication Info

Journal of the Chemical Society, Faraday Transactions, Volume 93, Issue 5, 1997, pages 781-789.

(c) [Journal of the Chemical Society, Faraday Transaction] [1997], [Royal Society of Chemistry].

Garashchuk, S., Grossmann,F. \& Tannor,D.(1997). Semiclassical approach to the hydrogen-exchange reaction- Reactive and transition-state dynamics. Journal of the Chemical Society, Faraday Transaction, 93(5), 781-789.

This Article is brought to you by the Chemistry and Biochemistry, Department of at Scholar Commons. It has been accepted for inclusion in Faculty Publications by an authorized administrator of Scholar Commons. For more information, please contact digres@mailbox.sc.edu. 


\title{
Semiclassical approach to the hydrogen-exchange reaction
}

\section{Reactive and transition-state dynamics}

\author{
Sophya Garashchuk, ${ }^{a}$ Frank Grossmann ${ }^{b}$ and David Tannor ${ }^{c}$ \\ ${ }^{a}$ Department of Physics, Department of Chemistry and Biochemistry, University of Notre Dame, \\ Notre Dame, IN 46556, USA \\ ${ }^{b}$ Albert-Ludwigs-Universität, Fakultät für Physik, Hermann-Herder-Str. 3, 79104 Freiburg, \\ Germany \\ ${ }^{c}$ Department of Chemical Physics, Weizmann Institute of Science, Rehovot, 76100, Israel
}

\begin{abstract}
Scattering matrix elements and symmetric transition-state resonances for the collinear $\mathrm{H}_{2}+\mathrm{H} \rightarrow \mathrm{H}+\mathrm{H}_{2}$ reaction are obtained using a time-dependent approach. The correlation function between reactant channel wavepackets and product channel wavepackets is used to determine the $S$-matrix elements. In a similar fashion, autocorrelation functions are used to extract the positions and widths of transition-state resonances. The time propagation of the wavepackets is performed by the improved semiclassical frozen Gaussian method of Herman and Kluk, which is an initial value, uniformly converged method. The agreement between the quantum and semiclassical results is far better than that obtained previously for this system by other semiclassical methods.
\end{abstract}

\section{Introduction}

The semiclassical limit of quantum mechanics has been a subject of great interest since the foundation of quantum mechanics. The possibility of describing quantum effects, such as interference and tunnelling, using classical trajectories is of both fundamental interest and great potential utility.

There exist a wide variety of semiclassical methods. The starting point for much of the subsequent work is the van Vleck propagator, which arises from a stationary-phase approximation to the exact quantum propagator within the path integral formulation. The van Vleck propagator expresses the transition probability from $x$ to $x^{\prime}$ in time $t$ in terms of a sum over classical paths, each weighted by a phase factor involving the classical action. It was refined many years later by Gutzwiller, who pointed out the need for an additional phase factor involving the Maslov index, to ensure the correct phase of the propagator after it passes through conjugate points. ${ }^{1-3}$ The numerical implementation of the van Vleck-Gutzwiller (VVG) propagator requires a search for classical trajectories with fixed initial and final position (or momentum) and a fixed propagation time. This is a doubleended boundary value problem, and requires extensive phase space sampling in order to find all such root trajectories. Even then, there are additional problems with coalescing root trajectories and divergences. Nevertheless, the VVG propagator has been successfully used for solving the dynamics of the stadium billiard, ${ }^{4}$ the Coulomb potential, ${ }^{5}$ the Rydberg atom $^{6,7}$ and scattering from the 1D Eckart barrier. ${ }^{8}$

Another family of semiclassical methods may be obtained from the VVG propagator by changing independent variables from the final position to the initial momentum, the so-called initial value representation. ${ }^{9-13}$ These methods avoid the need for a root search, but instead require the propagation of all initial conditions.

The thawed and frozen Gaussian wavepacket approaches, due to Heller, ${ }^{14,15}$ may be considered initial value methods. Strictly speaking, the thawed Gaussian ${ }^{14}$ method is semiclassical, representing an asymptotic solution of the time-dependent Schrödinger equation in the limit $\hbar \rightarrow 0$, while the frozen Gaussian method (FGA) ${ }^{15}$ is not. Both the thawed and frozen
Gaussian methods are easy to implement, but they are generally applicable only for short times or for potentials close to harmonic.

Subsequent to Heller's original work, Herman and Kluk ${ }^{16}$ (HK) put the frozen Gaussian method on a rigorous semiclassical footing by inserting an overcomplete set of Gaussians into the VVG propagator and requiring the correct asymptotic behaviour in the limit $\hbar \rightarrow 0$. In doing so, they discovered a complex prefactor, the analague of the van Vleck determinant, which was missing in the original formulation of Heller. ${ }^{15}$ Recently, Kay has rederived the HK propagator as a member of a family of semiclassical initial value propagators, and shown that the use of Gaussians in the method frees it from problems with caustic singularities that are present in the usual van Vleck formulation. ${ }^{17}$ The method has been applied recently, with impressive results, to a number of model problems. ${ }^{18-25}$

One of the great challenges of semiclassical methods has been the accurate calculation of reactive scattering probabilities. Previous semiclassical studies of reactive scattering have focussed on the collinear $\mathrm{H}+\mathrm{H}_{2}$ reaction. With one exception, ${ }^{26}$ these previous studies did not reproduce any of the resonances on this system and diverged in the energy region close to the barrier top $(0.6-0.8 \mathrm{eV}) .{ }^{26-28}$ Studies using frozen Gaussians on this system did not diverge, but were of limited accuracy and restricted to low energies. ${ }^{29,30}$ Two recent developments make it worthwhile to re-examine the use of semiclassical methods for reactive scattering. The first is the high quality of the recent results obtained using the HK method for non-reactive systems. The second is a recent wavepacket correlation function formulation of reactive scattering ${ }^{31-33}$ which treats reactants and products on the same footing, and potentially eliminates much of the difficulty associated with long time propagation. In this paper we test the HK method on the collinear $\mathrm{H}+\mathrm{H}_{2}$ reaction, using the wavepacket correlation function approach.

Apart from the reactive dynamics, transition-state dynamics of the $\mathrm{H}+\mathrm{H}_{2}$ system displays considerable complexity also, and leads to the formation of quantum mechanical resonances. Traditionally, the calculation of resonance energies and widths is performed in a time-independent framework. ${ }^{34}$ 
Nevertheless, much progress has been made recently in extracting resonance properties from wavepacket propagation for different model systems. Sadeghi and Skodje, for example, have studied transition-state resonance in the collinear $\mathrm{H}$ $+\mathrm{H}_{2}{ }^{35}$ and the isotopically substituted $\mathrm{D}+\mathrm{H}_{2}{ }^{36}$ reactions. Resonances in other systems have been studied in a timedependent framework by several authors. Isele et $a l^{37}$ have investigated the Rosen Thiele ABA model of two kinetically coupled Morse oscillators and Dai and Zhang calculated bound-state and resonance energies of $\mathrm{HO}_{2} \cdot{ }^{38}$ All these authors were solving the time-dependent Schrödinger equation using a split operator FFT method. ${ }^{39}$ Spectra and (resonance) eigenstates have been calculated by Fourier transformation of the dynamics. It has turned out that, for moderate accuracy requirements, the time-dependent method is a very fast and reliable computational tool for calculating resonance properties. Furthermore, doing the time propagation semiclassically, storage requirements are minimal, because the classical trajectories which enter the calculation of wavefunctions or correlation functions need not be stored and, for correlation functions, we do not even need to set up a grid for the calculation of the overlap, because in the case of a Gaussian initial wavefunction this integral can be done analytically.

To test further the predictive power of the semiclassical method it will be worthwhile to investigate the collinear $\mathrm{H}+\mathrm{H}_{2}$ exchange reaction with respect to its transition-state dynamics in the HK formalism. We thereby focus on the determination of resonance properties which are compared to full quantum calculations. It will be asked if the semiclassical method can reliably produce the positions and the widths of the symmetric transition-state resonances which have been determined previously fully quantum mechanically using a different potential surface. ${ }^{35}$ This will be a stringent test case for a semiclassical method because the system consists of the lightest atoms and therefore we are further away from the $\hbar \rightarrow 0$ limit than for a heavy-atom system. Semiclassical periodic-orbit-type investigations of resonances have been performed in non-generic systems like the three disc problem ${ }^{40}$ and for the dissociation of $\mathrm{HgI}_{2}{ }^{41}$ which shows Smale horseshoe-type behaviour in its classical dynamics at high energies. We want to stress that the straightforward implementation of semiclassical propagation in the time domain does not require the search for periodic orbits, however. Furthermore, the independence of the timedependent semiclassical approach from the underlying dynamics is very advantageous if one wants to study generic molecular systems. ${ }^{13}$ As will be shown, time-dependent semiclassics is readily applicable even for the calculations of such subtle quantities as resonance widths in these systems, and it is a promising tool for the investigation of even more complex problems than the one considered here.

The paper is organized as follows. In Section 2, we will give a sketch of the semiclassical methodology based on Herman and Kluk's version of the FGA. Section 3 contains the correlation function approach to the reactive scattering problem and Section 4 the corresponding formulation for the transition-state resonances. In Section 5 we present and discuss the numerical results for both problems. Finally, we will give conclusions and an outlook on possible future developments.

\section{Herman-Kluk propagator}

The semiclassical propagator, suggested by Herman and Kluk (HK), in $N$ dimensions is:

$$
\begin{aligned}
K^{\mathrm{sc}}\left(\boldsymbol{x}^{\prime}, t ; \boldsymbol{x}, 0\right)= & \frac{1}{(2 \pi \hbar)^{N}} \iint \mathrm{d} \boldsymbol{p}_{0} \mathrm{~d} \boldsymbol{q}_{0} R_{p q t} \exp \left(\imath S_{p q t} / \hbar\right) g_{\gamma} \\
& \times\left(\boldsymbol{q}_{t}, \boldsymbol{p}_{t}, \boldsymbol{x}^{\prime}\right) g_{\gamma}^{*}\left(\boldsymbol{q}_{0}, \boldsymbol{p}_{0}, \boldsymbol{x}\right)
\end{aligned}
$$

where

$$
g_{\gamma}\left(\boldsymbol{q}_{t}, \boldsymbol{p}_{t}, \boldsymbol{x}\right)=\left(\frac{\gamma}{\pi}\right)^{N / 4} \exp \left[-\frac{\gamma}{2}\left(\boldsymbol{x}-\boldsymbol{q}_{t}\right)^{2}+\frac{l}{\hbar} \boldsymbol{p}_{t} \cdot\left(\boldsymbol{x}-\boldsymbol{q}_{t}\right)\right]
$$

is a complex Gaussian,

$$
S_{p q t}=\int_{0}^{t}\left[\boldsymbol{p}_{t^{\prime}} \cdot \dot{\boldsymbol{q}}_{t^{\prime}}-H\left(\boldsymbol{p}_{t^{\prime}}, \boldsymbol{q}_{t^{\prime}}, t^{\prime}\right)\right] \mathrm{d} t^{\prime}
$$

is the classical action, and

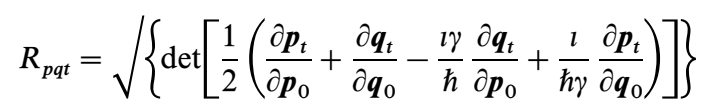

Here, $\boldsymbol{q}_{t}$ and $\boldsymbol{p}_{t}$ are the coordinates and momenta at time $t$ of a classical trajectory started with initial conditions $\boldsymbol{p}_{0}$ and $\boldsymbol{p}_{0}$ at time zero. The sign in eqn. (4) has to be chosen such that $R_{p q t}$ is a continuous function of time. ${ }^{17}$ The derivatives of a vector with respect to a vector in eqn. (4) imply a matrix of partial derivatives of components of one vector with respect to components of another vector. The integration goes over all initial values $\left(\boldsymbol{q}_{0}, \boldsymbol{p}_{0}\right)$.

The propagator is equal to the van Vleck form at all times, within the stationary-phase approximation. It is uniform, ${ }^{17}$ time reversible and unitary in the stationary-phase approximation. ${ }^{42}$

The general form for the semiclassical time correlation function of the two states $\psi_{\mathrm{A}}, \psi_{\mathrm{B}}$ is

$$
C_{\mathrm{AB}}^{\mathrm{cs}}(t)=\iint \mathrm{d} \boldsymbol{x} \mathrm{d} \boldsymbol{x}^{\prime} \psi_{\mathrm{B}}^{*}\left(\boldsymbol{x}^{\prime}, 0\right) K^{\mathrm{sc}}\left(\boldsymbol{x}^{\prime}, t ; \boldsymbol{x}, 0\right) \psi_{\mathrm{A}}(\boldsymbol{x}, 0)
$$

Substituting in the HK expression for the propagator leads to

$$
\begin{aligned}
C_{\mathrm{AB}}^{\mathrm{sc}}(t)= & \iint \mathrm{d} \boldsymbol{x} \mathrm{d} \boldsymbol{x}^{\prime} \psi_{\mathrm{B}}^{*}\left(\boldsymbol{x}^{\prime}, 0\right) \iint \frac{\mathrm{d} \boldsymbol{p}_{0} \mathrm{~d} \boldsymbol{q}_{0}}{(2 \pi \hbar)^{N}} R_{p q t} \exp \left(\frac{l S_{p q t}}{\hbar}\right) g_{\gamma} \\
& \times\left(\boldsymbol{q}_{t}, \boldsymbol{p}_{t}, \boldsymbol{x}^{\prime}\right) g_{\gamma}^{*}\left(\boldsymbol{q}_{0}, \boldsymbol{p}_{0}, \boldsymbol{x}\right) \psi_{\mathrm{A}}(\boldsymbol{x}, 0)
\end{aligned}
$$

which will be used in the subsequent sections.

\section{Correlation function formulation of the collinear hydrogen-exchange reaction}

We consider the collinear hydrogen-exchange reaction $\mathrm{H}_{a}+\mathrm{H}_{b} \mathrm{H}_{c}(v) \rightarrow \mathrm{H}_{a} \mathrm{H}_{b}\left(v^{\prime}\right)+\mathrm{H}_{c}$, where $v$ and $v^{\prime}$ denote quantum numbers of the vibrational eigenstates for reactants and products, respectively. The dynamics is governed by the two-dimensional Hamiltonian which in bond coordinates takes the form

$$
H=\frac{p_{X}^{2}}{m_{\mathrm{H}}}-\frac{p_{X} p_{Y}}{m_{\mathrm{H}}}+\frac{p_{Y}^{2}}{m_{\mathrm{H}}}+V(X, Y)
$$

Here, $X$ is the distance between $\mathrm{H}_{a}$ and $\mathrm{H}_{b}$ and $Y$ is the distance between $\mathrm{H}_{b}$ and $\mathrm{H}_{c}$. The Wall-Porter potential surface $^{43}$ was used, because of its simple analytical form and because of the extensive amount of prior semiclassical and quasiclassical work on this potential. ${ }^{26-28}$ In the asymptotic regions $X \gg Y$ or $Y \gg X$ the potential reduces to a Morse potential in the vibrational coordinate, simplifying the construction of the initial and final states. The surface parameters are taken from ref. 26.

To compute the $S$-matrix elements we follow the method of Tannor and Weeks, ${ }^{31-33}$ which expresses the $S$-matrix element as the Fourier transform of a cross-correlation function between a wavepacket which correlates with reactants and one which correlates with products. In our treatment here, two wavepackets are defined, one in each of the asymp- 
totic regions of the potential. $\dagger$ The wavepacket in the reactant channel is taken to be a direct product of a Gaussian in the translation coordinate, $R_{\mathrm{A}}$, and the $v$ eigenstates of the Morse oscillator in the vibrational coordinate, $r_{\mathrm{A}}$ :

$$
\begin{aligned}
& \psi_{\mathrm{A}}^{v}\left(R_{\mathrm{A}}, r_{\mathrm{A}}\right)=\left(\frac{\gamma}{\pi}\right)^{1 / 4} \\
& \times \exp \left[-\frac{\gamma}{2}\left(R_{\mathrm{A}}-R_{\mathrm{A}}^{0}\right)^{2}+\frac{l}{\hbar} P_{\mathrm{A}}^{0}\left(R_{\mathrm{A}}-R_{\mathrm{A}}^{0}\right)\right] \chi_{v}\left(r_{\mathrm{A}}\right)
\end{aligned}
$$

where $R_{\mathrm{A}}$ is the distance between the atom $\mathrm{H}_{a}$ and the centre of the diatomic $\mathrm{H}_{b} \mathrm{H}_{c}$, while $r_{\mathrm{A}}$ is the distance between $\mathrm{H}_{b}$ and $\mathrm{H}_{c}$ in the diatomic. Similarly, the wavepacket in the product channel is taken to be a direct product of a Gaussian in the translational coordinate $R_{\mathrm{B}}$ and the $v^{\prime}$ eigenstate of the Morse oscillator in the vibrational coordinate $r_{\mathrm{B}}$ :

$$
\begin{aligned}
\psi_{\mathrm{B}}^{v^{\prime}}\left(R_{\mathrm{B}}, r_{\mathrm{B}}\right) & =\left(\frac{\gamma}{\pi}\right)^{1 / 4} \\
& \times \exp \left[-\frac{\gamma}{2}\left(R_{\mathrm{B}}-R_{\mathrm{B}}^{0}\right)^{2}+\frac{l}{\hbar} P_{\mathrm{B}}^{0}\left(R_{\mathrm{B}}-R_{\mathrm{B}}^{0}\right)\right] \chi_{v^{\prime}}\left(r_{\mathrm{B}}\right)
\end{aligned}
$$

where $R_{\mathrm{B}}$ is the distance between the atom $H_{c}$ and the centre of the diatomic $\mathrm{H}_{a} \mathrm{H}_{b}$, and $r_{\mathrm{B}}$ is the distance between $\mathrm{H}_{a}$ and $\mathrm{H}_{b}$ in the diatomic.

Substituting eqn. (7) and (8) into eqn. (5), we obtain:

$$
C_{v v^{\prime}}^{\mathrm{sc}}(t)=\left(\frac{1}{2 \pi \hbar}\right)^{N} \iint \mathrm{d} \boldsymbol{p}_{0} \mathrm{~d} \boldsymbol{q}_{0} b_{p q t}^{*} a_{p q t} R_{p q t} \exp \left(\imath S_{p q t} / \hbar-c_{p q t}\right)
$$

where

$$
\begin{gathered}
a_{p q t}=\int \mathrm{d} r_{\mathrm{A}} g_{\gamma}^{*}\left(q_{0}, p_{0}, r_{\mathrm{A}}\right) \chi_{\mathrm{A}}\left(r_{\mathrm{A}}, 0\right) \\
b_{p q t}=\int \mathrm{d} r_{\mathrm{B}} g_{\gamma}^{*}\left(q_{t}, p_{t}, r_{\mathrm{B}}\right) \chi_{\mathrm{B}}\left(r_{\mathrm{B}}, 0\right) \\
c_{p q t}=\frac{\gamma}{4}\left[\left(Q_{t}-\bar{R}_{\mathrm{B}}^{0}\right)^{2}+\left(Q_{0}-R_{\mathrm{A}}^{0}\right)^{2}\right] \\
+\frac{l}{2 \hbar}\left(P_{t}+\bar{P}_{\mathrm{B}}^{0}\right)\left(Q_{t}-\bar{R}_{\mathrm{B}}^{0}\right)+\frac{1}{4 \gamma}\left[\left(P_{t}-\bar{P}_{\mathrm{B}}^{0}\right)^{2}\right. \\
\left.+\left(P_{0}-P_{\mathrm{A}}^{0}\right)^{2}\right]-\frac{l}{2 \hbar}\left(P_{0}+P_{\mathrm{A}}\right)\left(Q_{0}-Q_{\mathrm{A}}^{0}\right)
\end{gathered}
$$

The integration $\mathrm{d} \boldsymbol{p}_{0} \mathrm{~d} \boldsymbol{q}_{0}$ is performed over the Jacobi coordinates of reactants and their conjugate momenta, i.e. phasespace variables $\left(Q_{0}, P_{0}\right)$ and $\left(q_{0}, p_{0}\right)$ are introduced, associated with $R_{\mathrm{A}}$ and $r_{\mathrm{A}}$, respectively. Thus, $\mathrm{d} \boldsymbol{p}_{0} \mathrm{~d} \boldsymbol{q}_{0}=$ $\mathrm{d} P_{0} \mathrm{~d} Q_{0} \mathrm{~d} p_{0} \mathrm{~d} q_{0}$. The parameters $\left(\bar{R}_{\mathrm{B}}^{0}, \bar{P}_{\mathrm{B}}^{0}\right)$ are the parameters $\left(R_{\mathrm{B}}^{0}, P_{\mathrm{B}}^{0}\right)$ of the final state $\mathrm{B}$ defined by eqn. (8), expressed in coordinates $\left(Q_{0}, P_{0}\right)$.

Fourier transformation of the correlation function (9) provides the desired $S$-matrix element:

$$
S_{v v^{\prime}}(E)=\frac{\hbar \sqrt{ }\left(\left|p_{v^{\prime}}^{\prime} \| p_{v}\right|\right.}{2 \pi \eta^{*}\left(p_{v^{\prime}}^{\prime}\right) \eta\left(p_{v}\right)} \int_{-\infty}^{+\infty} \mathrm{d} t \exp \left(\frac{l E t}{\hbar}\right) C_{v v^{\prime}}(t)
$$

where $E$ is the total energy, $p_{v}=\sqrt{ }\left[2 m\left(E-E_{v}\right)\right]$ and $p_{v^{\prime}}^{\prime}=$ $\sqrt{ }\left[2 m\left(E-E_{v^{\prime}}\right)\right]$. The energy expansion coefficients $\eta_{v}$ and $\eta_{v^{\prime}}$ are the Fourier transforms of the initial Gaussians in the translational coordinates for the reactants and products, respectively, as defined above in eqn. (7) and (8)..$^{31,32}$

It is worth emphasizing that using the correlation function approach to scattering we obtain $S$-matrix elements for the specific initial and final internal quantum numbers over a

$\dagger$ Möller states were not used here, since the computation of the correlation function using the semiclassical wavefunction would require much greater numerical effort than the direct computation of the correlation function, which is all that is needed. wide, continuous range of energies by propagating a single wavepacket.

\section{Correlation function formulation for the transition-state resonances}

The symmetric transition-state resonances can be extracted from the dynamics of symmetric Gaussians, centred along the symmetric stretch of the $\mathrm{H}+\mathrm{H}_{2}$ potential. In order to keep the notation simple we specialize to the case of Gaussian wavepackets with the same width parameter $\gamma$ as in eqn. (2)

$$
\Psi_{\mathrm{A}}(\boldsymbol{x}, 0)=\left(\frac{\gamma}{\pi}\right)^{N / 4} \exp \left\{-\frac{\gamma}{2}\left(\boldsymbol{x}-\boldsymbol{q}_{\mathrm{A}}\right)^{2}+\frac{l}{\hbar} \boldsymbol{p}_{\mathrm{A}} \cdot\left(\boldsymbol{x}-\boldsymbol{q}_{\mathrm{A}}\right)\right\}
$$

with centre position $\boldsymbol{q}_{\mathrm{A}}$ and centre momentum $\boldsymbol{p}_{\mathrm{A}}$ as initial states for our dynamics.

For the extraction of transition-state resonance properties in a time-dependent fashion we again need correlation functions. The semiclassical correlation function of the form given in eqn. (5) will be employed in the following. In order to evaluate this expression we first calculate analytically the overlap of the initial Gaussian in eqn. (14) with the coherent state

$$
\begin{aligned}
\int \mathrm{d} \boldsymbol{x} g_{\gamma}^{*}\left(\boldsymbol{p}_{0}, \boldsymbol{q}_{0}, \boldsymbol{x}\right) \Psi_{\mathrm{A}}(\boldsymbol{x}, 0)=\exp \left\{-\frac{\gamma}{4}\left(\boldsymbol{q}_{0}-\boldsymbol{q}_{\mathrm{A}}\right)^{2}\right. \\
\left.+\frac{l}{2 \hbar}\left(\boldsymbol{q}_{0}-\boldsymbol{q}_{\mathrm{A}}\right)\left(\boldsymbol{p}_{0}+\boldsymbol{p}_{\mathrm{A}}\right)-\frac{1}{4 \gamma \hbar^{2}}\left(\boldsymbol{p}_{0}-\boldsymbol{p}_{\mathrm{A}}\right)^{2}\right\}
\end{aligned}
$$

Analogously, the overlap integral of $g_{\gamma}\left(\boldsymbol{p}_{t}, \boldsymbol{q}_{t}, \boldsymbol{x}\right)$ with a Gaussian $\Psi_{\mathrm{B}}(\boldsymbol{x})$ of the form of eqn. (14), can also be done analytically. Now that the integrations over $\boldsymbol{x}$ and $\boldsymbol{x}^{\prime}$ have been performed, the correlation function is finally represented as an integral over initial phase space. The $2 \mathrm{~N}$ dimensional integration over the variables $\boldsymbol{p}_{0}, \boldsymbol{q}_{0}$ will be performed numerically.

For the determination of resonance properties we will calculate autocorrelation functions, eqn. (5), and the corresponding half spectra ${ }^{35}$

$$
J(\omega) \sim\left|\int_{0}^{\infty} c_{\mathrm{AA}}(t) \exp (\imath \omega t) \mathrm{d} t\right|^{2}
$$

Comparing with full quantum calculations of the same quantities allows us to test the predictive power of the semiclassical theory for molecular systems with purely repulsive potentials.

\section{Numerical results and Discussion}

\section{A Reactive scattering case}

The initial and final wavepackets are chosen to be narrow in the translation coordinate and located close to the interaction region of the potential. The parameters in eqn. (7) and (8) are $\gamma=8, R_{\mathrm{A}}=R_{\mathrm{B}}=4.2, p_{\mathrm{A}}=-p_{\mathrm{B}}=-6.5$ for $v=0 \rightarrow v^{\prime}=0,1$ transitions and $\gamma=12.6, R_{\mathrm{A}}=R_{\mathrm{B}}=4.0, p_{\mathrm{A}}=-p_{\mathrm{B}}=-6.5$ for $v=0 \rightarrow v^{\prime}=2$ transition (in $\hbar=1, m_{\mathrm{H}} / 2=1, a_{0}=1$ units). The energy expansion coefficients are non-zero for the range of translational energies between 0.4 and $2.4 \mathrm{eV}$, and are centred at energies near the top of the barrier. The results are compared with quantum-mechanical results, obtained using the split operator propagation method ${ }^{39}$ and an absorbing potential. ${ }^{44}$ The total propagation time is taken to be $c a$. $5 \times 10^{4}$ atomic units (a.u.) in both the semiclassical and the quantum calculations.

We note, that, as in ref. 8, our semiclassical results show a dependence on the parameters of the initial and final wavepackets, despite the fact that the exact results are independent of these parameters. We have chosen the parameters here such that the initial and final wavepackets are quite narrow and located as close as possible to the barrier region. This cuts down on the propagation time necessary and thus reduces the intrinsic semiclassical error, as well as the numerical error. 
Note further, that the HK width parameter, $\gamma$, has been chosen equal to the width of the translational wavepacket. This was the same choice used in the transition-states resonances case, see Section 4, and in ref. 21 and 22 and has a heuristic motivation based on phase-space arguments. ${ }^{46}$

The integrals in eqn. (10) and (11) are performed using 25point Gauss-Hermite quadrature. ${ }^{47}$ The integration over the initial conditions in both translational and vibrational coordinates in eqn. (9) is done with a Gaussian weighted Monte Carlo sampling and the Sobol technique of generating quasirandom numbers, ${ }^{47}$ see also ref. 8 . The width of this Gaussian weight parameter is chosen equal to the zero point width in the vibrational coordinate and equal to the width of $\phi^{ \pm}$in the translational cordinate. The number of sampling points (which is the same as the number of classical trajectories) is $6 \times 10^{5}+$ The classical propagation is accomplished with the position Verlet algorithm. ${ }^{49}$

Fig. 1 compares the quantum and the semiclassical time correlation functions for $v=0 \rightarrow v^{\prime}=0$. The semiclassical correlation function is seen to agree with the quantum correlation function remarkably well, although some degradation in the agreement is observed at later times. Increasing the number of trajectories by a factor of 1.33 led to results of very similar quality, differing by, at most, $5 \%$ (and generally ca. $1 \%$ ) for the first half of the correlation function and $c a .15 \%$ for the second half, where the amplitude is much smaller. However, no change in either the frequency or phase of the recurrences was observed. Increasing the number of trajectories by an order of magnitude led to a significant decrease in the magnitude of the recurrences and increased the discrepancy with the exact results.

Fig. 2 compares the quantum and semiclassical transition probabilities $P_{v v^{\prime}}=\left|S_{v v^{\prime}}\right|^{2}$ for $v=0 \rightarrow v^{\prime}=0,1,2$. The semiclassical results computed with the HK method are a significant improvement over previous semiclassical and quasiclassical results on this system. ${ }^{26-28}$ With one exception, ${ }^{28}$ previous methods did not reproduce any of the resonances on this system and gave infinite results in the energy region close to the barrier top $(0.6-0.8 \mathrm{eV})$; the $\mathrm{HK}$ method

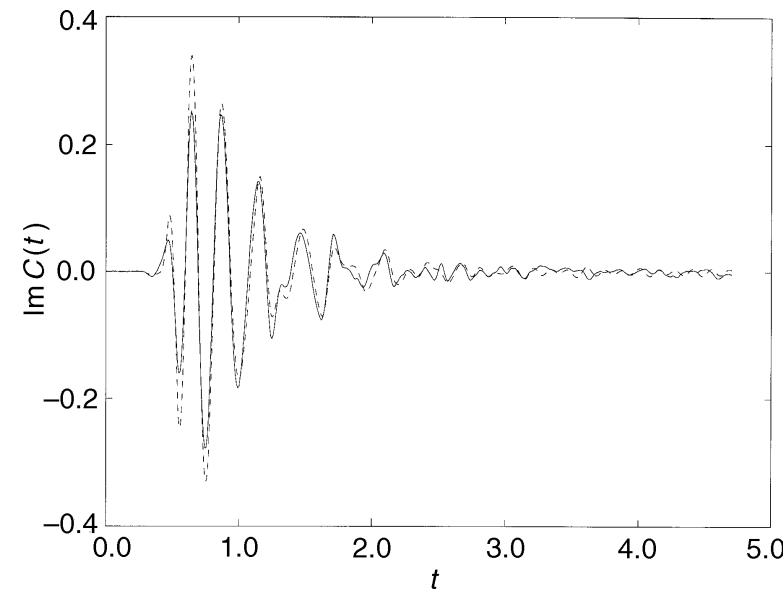

Fig. 1 Imaginary part of the cross-correlation function for collinear $\mathrm{H}_{2}(v=0)+\mathrm{H} \rightarrow \mathrm{H}+\mathrm{H}_{2}\left(v^{\prime}=0\right)$ obtained with quantum-mechanical $(\longrightarrow)$ and HK (- - - ) methods. Time is given in a.u.

$\ddagger$ We tried to use the 'cellular' version of the HK method suggested in ref. 18 and 21, but we found that, for this problem, the original formulation of the HK method performed better. The results of the 'cellularized' HK method were strongly dependent on the cell widths and not converged to the HK results for long times. Moreover, the need to perform matrix operations in the cellularized method eliminated the possible advantage due to the reduction in the number of classical trajectories.
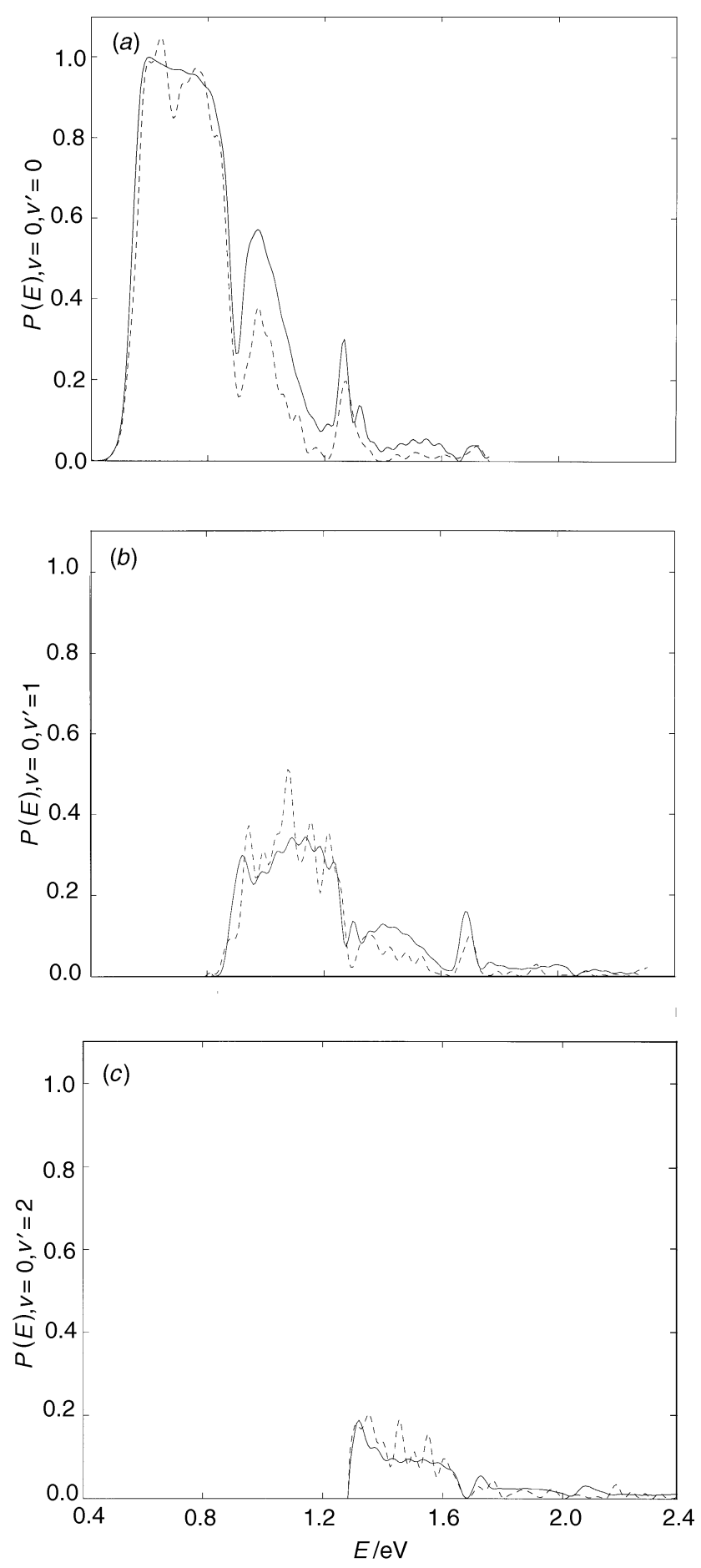

Fig. 2 Quantum-mechanical (- - ) and $\mathrm{HK}(----)$ reaction probabilities for collinear $\mathrm{H}_{2}(v)+\mathrm{H} \rightarrow \mathrm{H}+\mathrm{H}_{2}\left(v^{\prime}\right)$ for $(a) v=0 \rightarrow$ $v^{\prime}=0,(b) v=0 \rightarrow v^{\prime}=1,(c) v=0 \rightarrow v^{\prime}=2$

reproduces both the position and width of all the resonances and, being uniformly convergent, gives a finite result everywhere. Note, that the $\mathrm{HK}$ transition probability for $P_{00}$ exceeds unity for some energies. This reflects the fact that the HK method is unitary only in the limit of $\hbar \rightarrow 0$, not for finite values of $\hbar$. Most of the error in the transition probability can probably be attributed to the failure of the HK method to describe the long-time dynamics accurately, where contributions from tunnelling and above-barrier reflection are likely to be more important. It seems worthwhile to note that by inclusion of complex orbits, Maitra and Heller ${ }^{50}$ were able to improve on the shortcomings of the real trajectory approach for tunnelling in their study of the time-dependent semiclassical Green's function. 
Fig. 3 and 4 compare the quantum and the HK scattering reaction probability, summed over the final vibrational quantum number $v^{\prime}$ from 0 through 5. In Fig. 3 the initial quantum number is $v=0$, while in Fig. 4 the initial quantum number is $v=1$. Again the results are seen to be of semiquantitative accuracy. Fig. 5 checks time-reversal symmetry by comparing the reactive scattering probability for the $v=1 \rightarrow v=0$ transition and the $v=0 \rightarrow v=1$ transition.

A key question is how much of the discrepancy between the semiclassical and quantum results is arising from the numerical error in the Monte Carlo sampling, and whether the results can be improved by better sampling of initial conditions. Adequate Monte Carlo sampling has proved to be an extremely difficult computational problem whenever phase cancellation is involved, and has been the bottleneck to quantum and semiclassical methods based on path integration. An intriguing strategy for improving the sampling in the context of reactive scattering is to sample the classical trajectories from along a dividing surface in the interaction region (e.g. the top of the barrier) rather than from the asymptotic region; this eliminates from the sampling, any trajectories that never reach the dividing surface. Analogous ideas have greatly improved the sampling efficiency of trajectory-based rate calculations. ${ }^{51}$

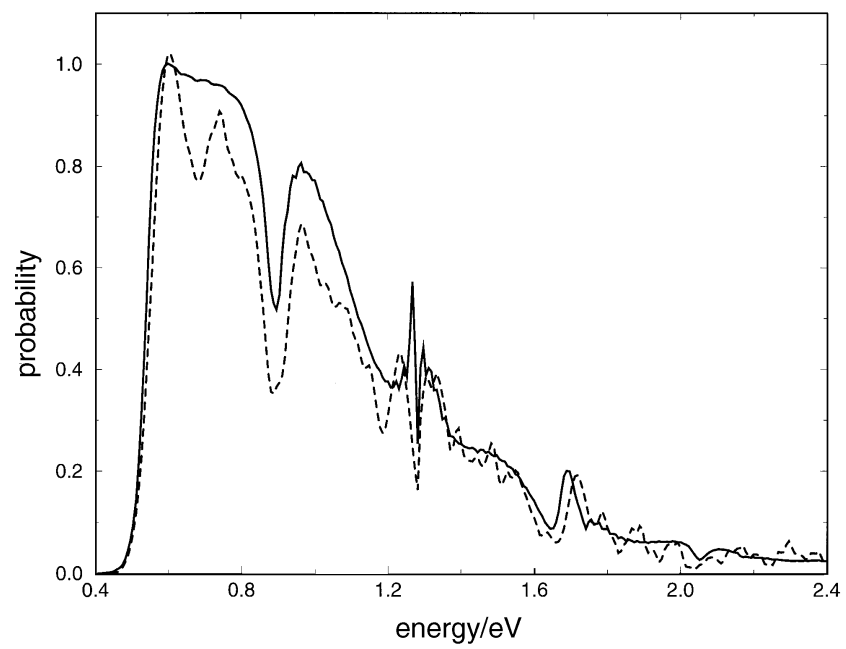

Fig. 3 Scattering reaction probability, $\sum_{v=0}^{5}\left|S_{0 v}\right|^{2}$, summed over the final vibrational quantum number $v^{\prime}$ from 0 through 5 . The initial quantum number is $v=0$. ( $\longrightarrow$ QM, $(---)$ HK.

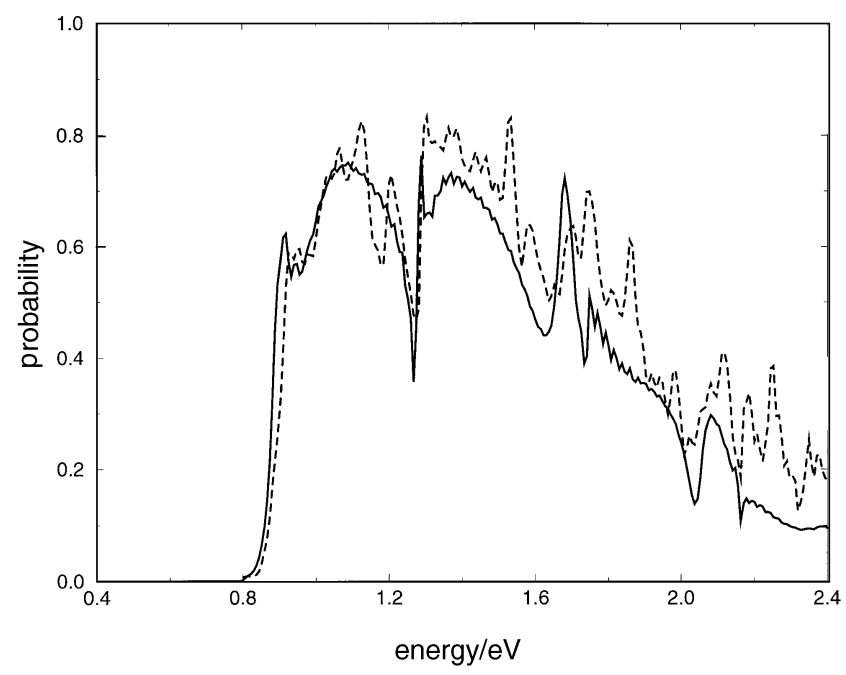

Fig. 4 Scattering reaction probability, $\sum_{v=0}^{5}\left|S_{1 v}\right|^{2}$, summed over the final vibrational quantum number $v^{\prime}$ from 0 through 5 . The initial quantum number is $v=1$. ( -$) \mathrm{QM},(---) \mathrm{HK}$.

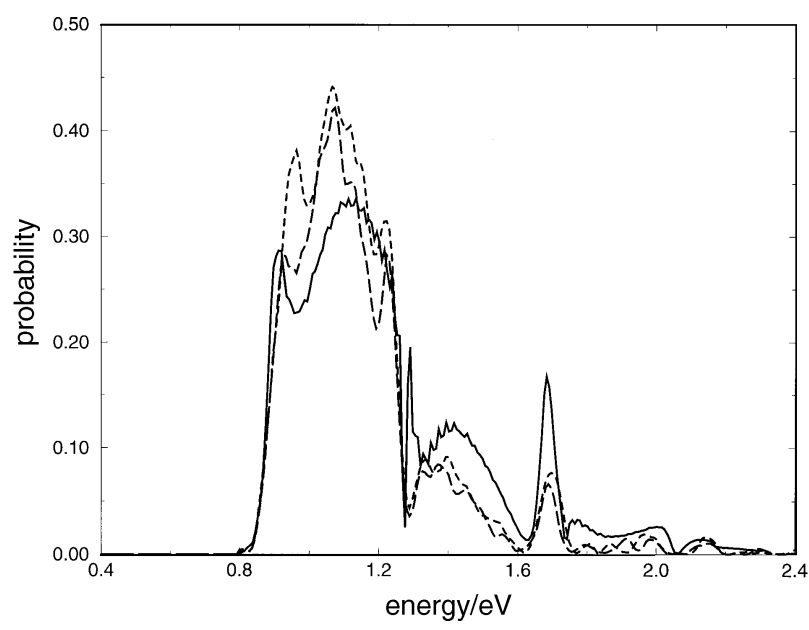

Fig. 5 Scattering reaction probability for the $v=0 \rightarrow v=1$ transition $(---)$ and $v=1 \rightarrow v=0$ transition $(--)$ calculated semiclassically, and $v=0 \rightarrow v=1$ transition ( $\longrightarrow$ calculated quantum mechanically. Detailed balance demands that the transition amplitudes $0 \rightarrow 1$ and $1 \rightarrow 0$ should be equal; the small discrepancy $(5 \%)$ can be attributed to numerical error in the Monte Carlo sampling of initial conditions.

We have, therefore, developed the following alternative implementation of eqn. (5). First a transformation of the initial variables is made, such that the variables $\left(\boldsymbol{q}_{0}, \boldsymbol{p}_{0}\right)$ become functions of the new variables $(\boldsymbol{q}, \boldsymbol{p})$ :

$$
\left\{\begin{array}{l}
\boldsymbol{q}_{0}=\boldsymbol{q}_{0}(\boldsymbol{q}, \boldsymbol{p}, \tau) \\
\boldsymbol{p}_{0}=\boldsymbol{p}_{0}(\boldsymbol{q}, \boldsymbol{p}, \tau)
\end{array}\right.
$$

with time $\tau$ being fixed. Then eqn. (5) can be rewritten as:

$$
C_{\mathrm{AB}}(t)=\iint \mathrm{d} \boldsymbol{p}_{0} \mathrm{~d} \boldsymbol{q}_{0} f\left(\boldsymbol{p}_{0}, \boldsymbol{q}_{0}, t\right)=\iint \mathrm{d} \boldsymbol{p} \mathrm{d} \boldsymbol{q} F(\boldsymbol{p}, \boldsymbol{q}, t)\left|J_{1}\right|
$$

where the function $f\left(\boldsymbol{p}_{0}, \boldsymbol{q}_{0}, t\right)$ denotes the integrand of eqn. (5) and $F(\boldsymbol{p}, \boldsymbol{q}, t)$ is the transformed integrand. Note, that the transformation is canonical, and its Jacobian $\left|J_{1}\right|=1$. The physical interpretation of eqn. (17) is that now trajectories are propagated forward in time $t-\tau$ towards products and backward in time $\tau$ towards reactants, contributing to the correlation function at time $t$.

We now shift perspectives and view the parameter $\tau$ as an independent variable. Note that, since all reactive trajectories must cross the dividing surface (the transition state), the variable $\tau$ can be used to replace the coordinate perpendicular to the transition state as an independent variable. Thus, the second transformation [assuming a 2D Hamiltonian $H(\boldsymbol{q}, \boldsymbol{p})$ $=H\left(p_{1}, p_{2}, q_{1}, q_{2}\right)$, where $q_{1}$ is the coordinate parallel to the dividing surface and $q_{2}$ is perpendicular to it] takes the form:

$$
\begin{cases}q_{1}=q_{1} & q_{2}=q_{2}\left(q_{1}, \tau, p_{1}, p_{2} ; q_{2}^{*}\right) \\ p_{1}=p_{1} & p_{2}=p_{2}\end{cases}
$$

Here, $q_{2}^{*}$ is a fixed coordinate, representing a dividing value in the coordinate space, understood to be located at the transition state. The Jacobian of this transformation is

$$
\begin{aligned}
J_{2} & =\left(\begin{array}{llll}
\partial q_{1} / \partial q_{1} & \mathrm{~d} q_{1} / \mathrm{d} \tau & \partial q_{1} / \partial p_{1} & \partial q_{1} / \partial p_{2} \\
\partial q_{2} / \partial q_{1} & \mathrm{~d} q_{2} / \mathrm{d} \tau & \partial q_{2} / \partial p_{1} & \partial q_{2} / \partial p_{2} \\
\partial p_{1} / \partial q_{1} & \mathrm{~d} p_{1} / \mathrm{d} \tau & \partial p_{1} / \partial p_{1} & \partial p_{1} / \partial p_{2} \\
\partial p_{2} / \partial q_{1} & \mathrm{~d} p_{2} / \mathrm{d} \tau & \partial p_{2} / \partial p_{1} & \partial p_{2} / \partial p_{2}
\end{array}\right) \\
& =\left(\begin{array}{cccc}
1 & 0 & 0 & 0 \\
\partial q_{2} / \partial q_{1} & \partial H / \partial p_{2} & \partial q_{2} / \partial p_{1} & \partial q_{2} / \partial p_{2} \\
0 & 0 & 1 & 0 \\
0 & 0 & 0 & 1
\end{array}\right)
\end{aligned}
$$


The determinant of the Jacobian is given by:

$$
\left|J_{2}\right|=\frac{\partial H}{\partial p_{2}}
$$

Thus, for the $\mathrm{H}_{2}+\mathrm{H}$ Hamiltonian (6) (which becomes uncoupled in momenta when transformed into Jacobi coordinates $^{52}$ ), the Jacobian from eqn. (19) takes the form $\left|J_{2}\right|=p_{2} /\left(m_{\mathrm{H}} / \sqrt{ } 3\right)$. The final expression for the correlation function is

$$
C_{\mathrm{AB}}(t)=\iint \mathrm{d} p_{1} \mathrm{~d} p_{2} \mathrm{~d} q_{1} \mathrm{~d} \tau \frac{p_{2}}{m_{\mathrm{H}} / \sqrt{3}} \bar{F}\left(p_{1}, p_{2}, q_{1}, t, q_{2}^{*}\right)
$$

where $\bar{F}\left(p_{1}, p_{2}, q_{1}, t, q_{2}^{*}\right)$ is the integrand of eqn. (17) after the second transformation. Thus, the contribution of each trajectory to the correlation function is proportional to the momentum component perpendicular to the dividing surface. This result is again reminiscent of the method of reactive flux in trajectory calculations of rate constants, where trajectories contribute proportionally to their momentum perpendicular to the dividing surface. ${ }^{53}$ Note, that both of the above transformations are symmetric with respect to reactants and products, a principle central to the whole correlation function approach.

Fig. 6 shows a comparison between asymptotic sampling and transition state sampling for the 1D Eckart barrier. The parameters of the system, as well as the initial and final wavepackets, are given in ref. 8. In the asymptotic sampling, the phase space in the asymptotic region is sampled with a Gaussian-weighted Monte Carlo procedure; non-reactive trajectories are not propagated. In the transition-state sampling, the momentum at the dividing surface is sampled uniformly. The improvement in the calculated transmission probability using transition-state sampling is dramatic: the spurious oscillations in the transmission coefficient are virtually completely removed, and the agreement with the exact value of unity at high energies is orders of magnitude better than before. Moreover, the computation for the transition-state sampling is 20 times faster than for the asymptotic sampling. We attribute the gain in numerical efficiency to 'recycling' the same classical trajectories, i.e. having them contribute to the correlation function at multiple times, as well as to better representation of the trajectories with high and low energies. The transition probability computed with the standard VVG method is also depicted in Fig. 6. The computation time for the VVG method is 1.5 times longer than the HK method with asymptotic region

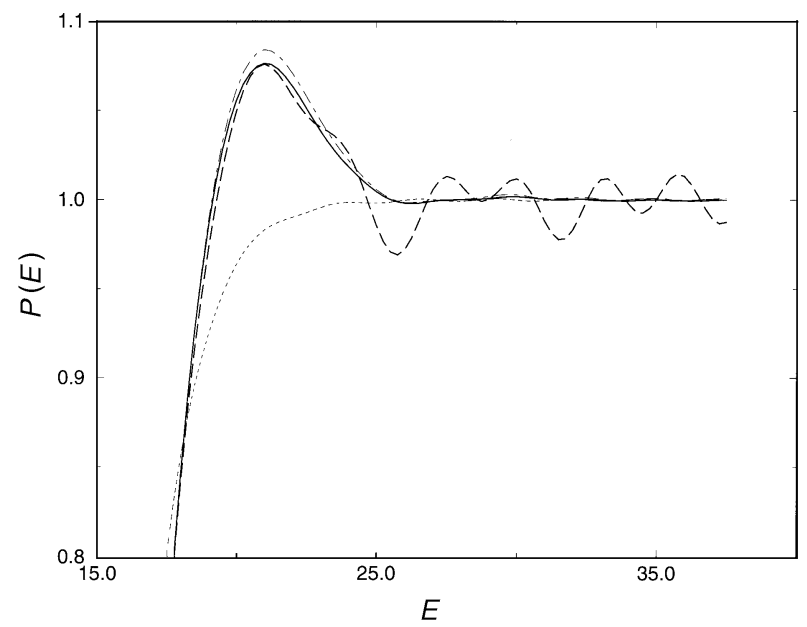

Fig. 6 Transmission coefficients for the 1D Eckart barrier. Quantum-mechanical $(\cdots \cdots \cdot)$, VVG method (-. - , HK asymptotic-state sampling method (- - ) and HK transition-state sampling method ( $\longrightarrow$ ). The energy of the top of the barrier is 16 a.u. Parameters of the initial and final wavepackets, in a.u., are $q_{\alpha}=-q_{\beta}=6, p_{\alpha}=p_{\beta}=6.5, \alpha=\beta=10$. and 30 times longer than the HK method with transition-state sampling. Note, that none of the three semiclassical methods really gives a quantitative description of tunnelling and above barrier reflection, resulting in transmission probabilities greater than unity for energies right above the top of the barrier. (The results of both ref. 8 and ref. 11 are plotted on a $\log$ scale. Therefore, a transmission probability of 1.07 is not inconsistent with these results. Recall also that the semiclassical results depend on the choice of $\phi^{ \pm}$, even though the exact quantum results do not.)

For the $\mathrm{H}_{2}+\mathrm{H}$ reaction, however, we found no improvement in either accuracy or efficiency with the transition-state sampling method. The dividing surface coordinate and the 2D momentum space were sampled with a uniform Monte Carlo procedure. The explanation may be that the efficiency gained by eliminating trajectories that never reach the dividing surface is somewhat compensated in transition-state sampling by the need to count the same trajectory multiple times as it crosses the dividing surface with different values of momentum.

\section{B Transition-state resonances case}

Before we present the results of our semiclassical calculations of transition-state resonances, let us briefly discuss the potential surface that will be studied. The analytical model potential which seems best suited for our purposes in this section is the semiempirical $\mathrm{H}+\mathrm{H}_{2}$ surface given by Porter and Karplus in the early sixties. ${ }^{54} \mathrm{~A}$ contour plot of this potential in bond coordinates is shown in Fig. 7. The PK potential is smooth for all values of the binding distances which are relevant for our study. The classical dynamics in the reactive scattering case has stochastic boundaries between reactive and non-reactive regions. We have observed similar boundaries for the case of a transition-state dynamics, see Fig. 8. Trajectories with the same energy show stochastic behaviour as to what side of the potential barrier (reactants or products) they will end up for long times. As can be seen in Fig. 8, for long times, trajectories which are close to periodic orbits of the system will survive and contribute considerably to the calculation of the autocorrelation function. Nevertheless, the time-dependent methodology used here has the advantage that periodic orbits need

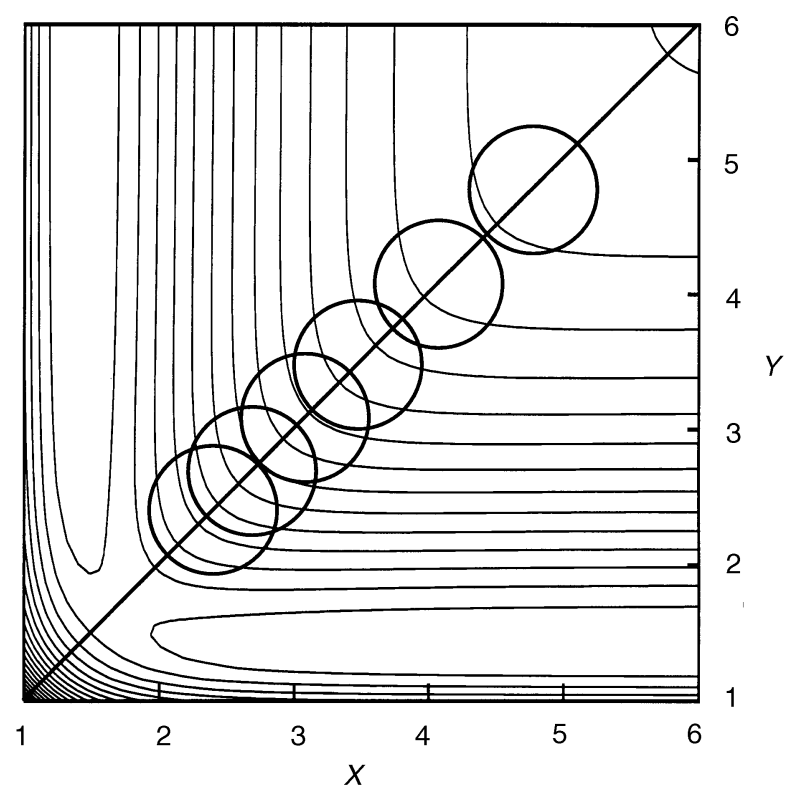

Fig. 7 Initial wavepackets centred along the symmetric stretch line are represented by circles (depicting the locus where the wavefunction is at $1 / e$ of its maximum value) displayed on top of the contour lines of the Porter-Karplus potential surface 

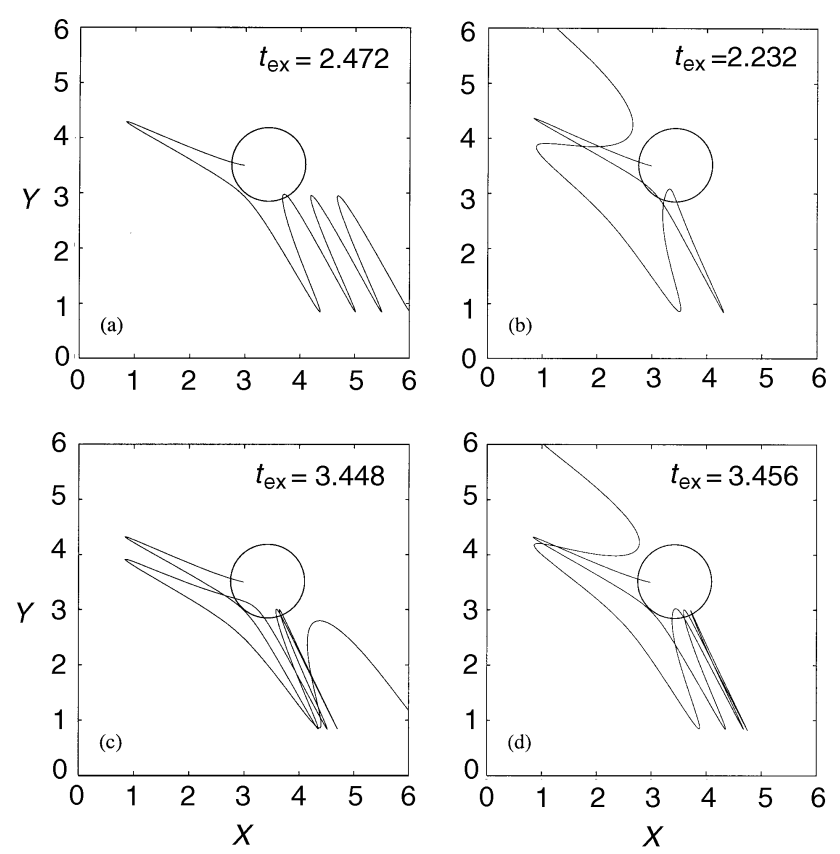

Fig. 8 Time evolution of four trajectories (a)-(d) starting at $(X=3$, $Y=3.5$ ) and with initial momenta on consecutive points of an ellipse of constant energy. The circles denote the locus where the positiondependent part of the exponent damping factor in the equation corresponding to eqn. (15) for the final state is at a value of $1 / e$. The exit times are given in units of $\mu a_{0}^{2} / \hbar$ and denote the times when the trajectories hit either $X=6$ or $Y=6$.

not be determined; one just has to solve initial value problems.

In the following, we will compare full time-dependent quantum calculations which have been done using the split operator FFT method ${ }^{39}$ with our time-dependent semiclassical calculations. The classical trajectories and their actions and stability information, which enter the semiclassical evaluation of correlation functions, have again been determined using the position-type Verlet method. It only requires one evaluation of the potential and its first and second derivatives per time step. For the results presented in the following we used $O\left(10^{7}\right)$ trajectories of which we only needed to propagate a small fraction to the very end. The major part of them quickly exited into one of the channels and no longer contributed to the calculation of the autocorrelation function. The remaining integration over initial phase space in eqn. (5) is finally done by employing a Monte Carlo scheme which is based on a Box-Müller algorithm and using standard pseudorandom numbers.

For the determination of autocorrelation functions, we have prepared six different initial wavepackets of the form given in eqn. (14) with initial centre parameters (measured in atomic units) along the symmetric stretch line of the potential surface ranging from $\boldsymbol{q}_{\mathrm{A}}=(2.4,2.4)$ to $\boldsymbol{q}_{\mathrm{A}}=(4.8,4.8)$ and zero initial momentum. The initial width parameter was kept at the value of $\gamma=9$ for all the wavepackets so that each of them has considerable overlap with two or three resonance states. These initial states are represented by circles depicted on the contour plot of the potential surface in Fig. 7. Using these wavepackets we were able to extract symmetric transition-state resonances.

Let us first give an example of how well the semiclassical propagation compares with the quantum one for a wavepacket that is centred so far out along the symmetric stretch line of the potential that it contains some of the energetically higher resonance states we are interested in. Fig. 9 shows a comparison of the absolute value of the semiclassical (dotted line) to the quantum (full line) autocorrelation function for an

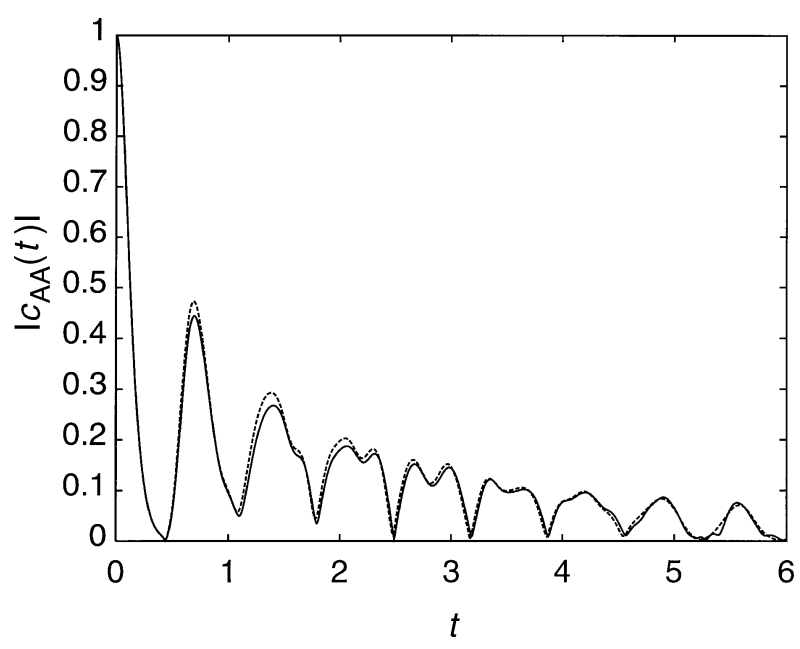

Fig. 9 Comparison of the absolute value of the semiclassical ) with the quantum mechanical (---) auto-correlation function for a Gaussian wavepacket initially centred around $(X=3.5$, $Y=3.5)$ on the symmetric stretch line of the $\mathrm{H}+\mathrm{H}_{2}$ system. The time $t$ is measured in units of $\mu a_{0}^{2} / \hbar$.

initial state with centre parameters $\boldsymbol{q}_{\mathrm{A}}=(3.5,3.5)$. It is very pleasing to see how the semiclassical correlation function represents the quantum behaviour for a time span of eight recurrences. In the case considered this amounts to approximately $130 \mathrm{fs}$ ( $\triangle t=1$ corresponds to $22 \mathrm{fs})$.

Spectra that have been extracted from the time series in Fig. 9 are displayed in Fig. 10. It is only because of a lack of convergence in the long time behaviour that the semiclassical line shape does not more exactly mirror the quantum result. From the spectra presented in Fig. 10 we extracted the properties of the two dominant resonances. By a fitting procedure of single peaks in the semiclassical and quantal spectra to a Lorentzian $^{35}$

$$
L(\omega)=\frac{h_{v}}{\left(\omega-\omega_{v}\right)^{2}+\left(\Gamma_{v}^{2} / 4 \hbar^{2}\right)}+C_{v}
$$

the positions $\omega_{v}$ can be determined with an accuracy of better than $1 \%$ and the widths $\Gamma_{v}$ have error bars of $c a .10 \%$ which come mainly from the difference between the actual line shape and a Lorentzian. Because of poor Monte Carlo statistics for long times, there are additional errors in the semiclassical widths. Nevertheless, the semiclassical results agree very well with the quantum mechanical ones as can be seen in Table 1

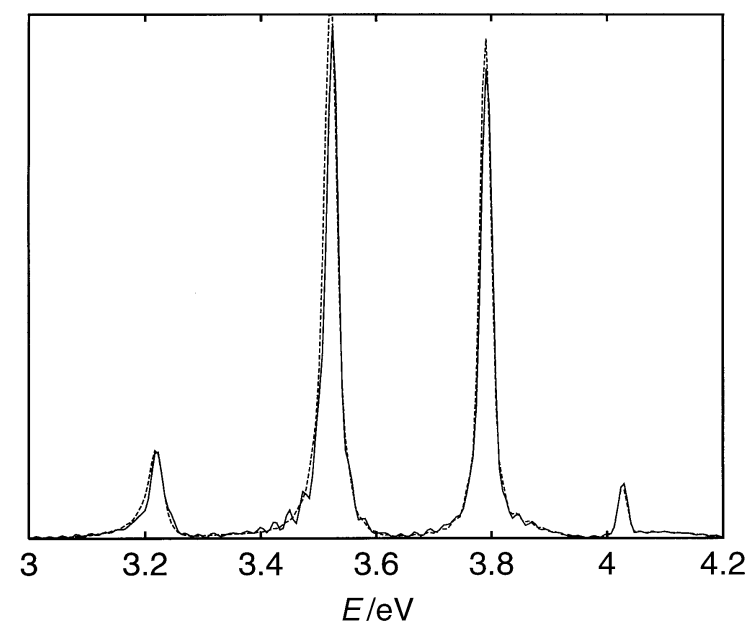

Fig. 10 Comparison of the peak structure of the semiclassical $(-)$ with the quantum mechanical $(----)$ half spectrum for the Gaussian wavepacket of Fig. 9 
Table 1 Quantum mechanical (QM) and semiclassical (SC) resonance properties for the collinear Porter-Karplus $\mathrm{H}+\mathrm{H}_{2}$ surface

\begin{tabular}{rccccc}
\hline$v$ & $E_{\mathrm{thr}}$ & $E_{v}^{\mathrm{QM}}$ & $E_{v}^{\mathrm{SC}}$ & $\tau_{v}^{\mathrm{QM}}$ & $\tau_{v}^{\mathrm{SC}}$ \\
\hline 1 & 0.795 & 0.881 & 0.884 & 28 & 18 \\
2 & 1.284 & 1.323 & 1.326 & 23 & 17 \\
3 & 1.741 & 1.750 & 1.753 & 16 & 15 \\
4 & 2.166 & 2.154 & 2.159 & 15 & 14 \\
5 & 2.588 & 2.540 & 2.544 & 16 & 16 \\
6 & 2.918 & 2.895 & 2.899 & 18 & 17 \\
7 & 3.246 & 3.227 & 3.231 & 21 & 20 \\
8 & 3.541 & 3.521 & 3.524 & 25 & 26 \\
9 & 3.804 & 3.790 & 3.792 & 33 & 29 \\
10 & 4.035 & 4.025 & 4.027 & 42 & 38 \\
11 & 4.233 & 4.229 & 4.231 & 55 & 53 \\
12 & 4.399 & 4.396 & 4.398 & 95 & 86 \\
13 & 4.533 & 4.532 & 4.532 & 142 & n.a. \\
14 & 4.634 & 4.635 & n.a. & n.a. & n.a. \\
\hline
\end{tabular}

The energies are given in $\mathrm{eV}$ and the widths $\Gamma$ by their corresponding lifetimes $\tau=0.6582 / \Gamma(\mathrm{eV})$ in fs. The thresholds for the $\mathrm{H}+\mathrm{H}_{2}(v)$ channels are given as $E_{\mathrm{thr}}$, the dissociation threshold is at $4.747 \mathrm{eV}$.

where we list fourteen symmetric transition-state resonances, labelled by the quantum number $v$. For the positions of the resonances we have extremely good agreement between the semiclassical and the quantal result. We want to remark that the resonances appear close to the vibrational thresholds $(v=1,2,3, \ldots)$, which are listed in Table 1 . It is very encouraging that the accuracy of the widths also compares for nine of the resonances to the numerical uncertainty which is inherent in their extraction. The fourteenth resonance is a very sharp peak at the high end of the spectrum which we did not investigate in any more detail. The accuracy of the semiclassical widths is analogous to that presented in Fig. 10 for resonances 8 and 9, except for the high- and low-energy resonances. For the low-energy resonances, which live close to the saddle point of the potential, we assume that non-classical effects such as e.g. tunnelling, might be responsible for the discrepancy of the results. The very high energy ones live too long to be described exactly semiclassically.

\section{Conclusion}

The time-dependent semiclassical method of Herman and Kluk was combined with a time correlation function approach to reactive scattering, and applied to the collinear hydrogen-exchange reaction. The extent of agreement with the quantum mechanical state-to-state transition probabilities shows a remarkable improvement over earlier studies which used other semiclassical approaches. In particular, for $v=0 \rightarrow v^{\prime}=0,1,2$ the results are everywhere finite; all the quantum resonances are obtained semiquantitatively; and there is no apparent degradation of accuracy for increasing $v^{\prime}$.

One would tend to suspect that the high-frequency oscillations in the semiclassical $S(E)$ originate from numerical error in the Monte Carlo sampling; however, we believe this is not the case. The Monte Carlo integration was implemented both from the asymptotic region in, and from the transition state region out, as well as with a variable number of sampling points. In all cases, the semiclassical results agreed with each other much more closely than with the quantum, suggesting that the error is intrinsic to the semiclassical approximation. Additional support for this conclusion is provided by the results on the 1D Eckart potential shown in Fig. 3. Those calculations were done by three different methods, each of which gave a transmission probability of 1.07 near the barrier top. Two of these methods (HK from the inside out, which samples initial conditions by quadrature, and van Vleck) are numerically converged to within $1 \%$, and hence the discrepancy from the quantum results must be inherent to the semiclassical approximation. Although the semiclassical and quantum Eckart $P(E)$ differ only in a single broad peak, as compared with the high-frequency oscillations seen in the $\mathrm{H}$ $+\mathrm{H}_{2} P(E) \mathrm{s}$, in both cases the discrepancies trace to the same source in the time domain; degradation of the semiclassical correlation function at long times. Thus, we tentatively conclude that most of the semiclassical/quantum discrepancy we see in $\mathrm{H}+\mathrm{H}_{2}$ is inherent to the semiclassical approximation.

Of course, it would be desirable to have complete numerical convergence of the semiclassical $\mathrm{H}+\mathrm{H}_{2}$ probabilities, to gauge unambiguously the semiclassical contribution to the error; but this was not possible. Increasing the number of Monte Carlo trajectories in $\mathrm{H}+\mathrm{H}_{2}$ by another order of magnitude led to a washing out of the recurrences in the interval 2000-4000 a.u., and an increase in the discrepancy with the quantum results. Numerical convergence was also attempted via quadrature (i.e. evenly spaced) sampling of initial conditions. This too was unsuccessful: the number of initial conditions required for convergence scales exponentially with the time interval, and the numerical effort required to obtain convergence on these timescales was prohibitive.

It should be noted that, in the time correlation function formulation, the semiclassical value of $S(E)$ depends on the choice of the Møller states, $\phi^{ \pm}$, despite the fact that the quantum $S$-matrix does not. We found, empirically, that choosing the Møller states to be quite narrow and located as close as possible to the barrier top gave the best agreement with the exact results. Presumably, this is because this choice minimizes the propagation time necessary, and concentrates the dynamics around the barrier top where a parabolic barrier, for which the HK method is exact, is a good description. $^{8-11}$ The fact that different choices of the Møller states had a larger effect on the results than different Monte Carlo implementations is a further indication that most of the discrepancy between our results and the quantum values is coming from intrinsic semiclassical error.

In principle, the agreement with the quantum mechanics could be improved further if one were to optimize the $\phi^{ \pm}$ states systematically and allow for non-Gaussian shapes. Furthermore, the HK width parameter $\gamma$ could, in principle, be optimized to improve agreement with the quantum mechanics. In practice, such an optimization risks becoming little more than an exercise in parameter fitting unless it leads to general, prescriptive ways for choosing the parameters for new systems a priori. However, the fact that these results leave some room for improvement is important in assessing the ultimate accuracy of which semiclassical methods might be capable.

The computer time required for the quantum mechanical calculation of a single $S$-matrix element is $c a$. 3-4 times less than for the semiclassical one. However, the CPU time required to generate the entire $S$-matrix (up to $v=5, v=5$ ) was less for the semiclassical calculation than for the quantum (ca. $12000 \mathrm{~s}$ cf. $15000 \mathrm{~s}$ ). This is because the semiclassical calculation evaluates the propagator $\left\langle x^{\prime}|\exp (-\imath H t / \hbar)| x\right\rangle$, which once calculated can then be used to generate the entire $S$ matrix. Moreover, the semiclassical method requires virtually no storage: the classical trajectories contribute to the correlation function in eqn. (9) independently, which is the advantage of the locality of classical mechanics. This is in contrast to the exact quantum mechanical methods, which are global and require storage of the multidimensional wavefunction on a grid. Such storage requirements grow exponentially with the number of dimensions, and become prohibitive for more than a few degrees of freedom.

By studying symmetric transition-state resonances of the same reaction, we demonstrated that it is within the reach of the semiclassical approximation to determine resonance widths to a reasonable degree of accuracy. There is no restriction concerning the type of system which can be studied in the HK formalism that has been used. Furthermore, periodic orbits need not be determined. The classical trajectories 
which contribute to the semiclassical correlation function have been calculated by a position-type Verlet method which has become a standard tool in large-scale molecular dynamics simulations and can easily be implemented for systems with many degrees of freedom. The need to propagate $O\left(10^{7}\right)$ trajectories for systems with two degrees of freedom seems disturbing. The positions of the resonances can, however, be determined semiclassically quite accurately with one order of magnitude fewer trajectories. The widths' accuracy deteriorates quite rapidly compared to the positions' accuracy, when using fewer trajectories.

Let us finally sum up the merits of the HK approach as compared with full quantum calculations. First, because of the locality of the classical dynamics, storage requirements are minimal and allow one to tackle systems with many degrees of freedom. Furthermore, as mentioned above, the time for constructing the full matrix was actually shorter using the semiclassical method than using a converged quantum mechanical method with the same formulation of the $S$-matrix. This is extremely encouraging, given the quite general experience that semiclassical methods are in fact slower than fully quantum methods. More importantly, however, a more favourable scaling behaviour of the computer time with dimensionality than in the quantum case might possibly result. This should open the door to calculations on much larger systems than have been possible to address previously. In fact, recent results show that the present semiclassical method can be converged for $\operatorname{Ar}_{6} \mathrm{I}^{-},{ }^{55}$ i.e. 15 coupled vibronic degrees of freedom, for which straightforward quantum scattering methods are clearly intractable. Lastly, we want to emphasize that the locality of classical dynamics also opens a way to understand quantum phenomena, ${ }^{13}$ which is very helpful for interpretational reasons. With an increasing understanding of the subtleties of the Monte Carlo method used for the complicated integrands studied here, this aspect will also add to the usefulness of a semiclassical approach like the one presented here.

Now that both reactive and transition-state resonances in the collinear $\mathrm{H}+\mathrm{H}_{2}$ system have been uncovered semiclassically, a long-standing goal in theoretical chemical physics has been reached. The quantum mechanical transition-state resonances, close to the vibrational thresholds, arise from the same physical origin as the resonances in the reactive scattering calculation. For a nice discussion of this point see e.g. ref. 56 .

We are grateful to Ken Kay and David Manolopoulos for helpful discussions, and for communicating their results prior to publication. This work was funded by the NSF and by the Deutsche Forschungsgemeinschaft through Sonderforschungsbereich 276 .

\section{References}

1 M. Gutzwiller, Chaos in Classical and Quantum Mechanics, Springer-Verlag, New York, 1990.

2 R. Littlejohn and J. Robbins, Phys. Rev. A, 1987, 36, 2953.

3 R. Littlejohn, Phys. Rev. Lett., 1988, 61, 2159.

4 S. Tomsovic and E. J. Heller, Phys. Rev. Lett., 1991, 67, 664; Phys. Rev. E, 1993, 47, 282.

5 I. Suárez Barnes, M. Nauenberg, M. Nockleby and S. Tomsovic, J. Phys. A, 1994, $27,3299$.

6 M. Mallalieu and C. Stroud, Phys. Rev. A, 1994, 49, 2329.
7 M. Mallalieu and C. Stroud, in Coherent State: Past, Present, and Future, World Scientific, Singapore, 1994.

8 F. Grossmann and E. J. Heller, Chem. Phys. Lett., 1995, 241, 45.

9 W. H. Miller, Adv. Chem. Phys., 1974, 25, 69.

10 W. H. Miller, J. Chem. Phys., 1991, 95, 9428.

11 S. Keshavamurthy and W. H. Miller, Chem. Phys. Lett., 1994, 218, 189.

12 E. J. Heller, J. Chem. Phys., 1991, 94, 2719.

13 M. A. Sepúlveda and F. Grossmann, Adv. Chem. Phys., 1996, 96, 191.

14 E. J. Heller, J. Chem. Phys.1975, 62, 1544.

15 E. J. Heller, J. Chem. Phys., 1981, 75, 2923.

16 M. F. Herman and E. Kluk, Chem. Phys., 1984, 91, 27.

17 K. G. Kay, J. Chem. Phys., 1994, 100, 4377.

18 K. G. Kay, J. Chem. Phys., 1994, 100, 4432.

19 K. G. Kay, J. Chem. Phys., 1994, 101, 2250.

20 E. Kluk, M. F. Herman and H. L. Davis, J. Chem. Phys., 1986, 84, 326.

21 A. Walton and D. Manolopoulos, Mol. Phys., 1996, 87, 961.

22 A. Walton and D. Manolopoulos, Chem. Phys. Lett., 1995, 244, 448.

23 F. Grossmann, Chem. Phys. Lett., 1996, 262, 470.

24 S. Garashchuk and D. Tannor, Chem. Phys. Lett., 1996, 262, 477.

25 For a different semiclassical initial value approach see B. W. Spath and W. H. Miller, Chem. Phys. Lett., 1996, 262, 486.

26 J. R. Stine and R. A. Marcus, Chem. Phys. Lett., 1974, 29, 575.

27 D. G. Truhlar and A. Kupperman, J. Chem. Phys., 1972, 56, 2232.

28 J. M. Bowman and A. Kupperman, J. Chem. Phys., 1973, 59, 6524.

29 R. T. Skodje and D. J. Truhler, J. Chem. Phys. 1984, 80, 3123.

30 J. D. Kress and A. E. DePristo, J. Chem. Phys., 1989, 89, 2886.

31 D. J. Tannor and D. E. Weeks, J. Chem. Phys., 1993, 98, 3884.

32 D. E. Weeks and D. J. Tannor, Chem. Phys. Lett., 1993, 207, 301

33 D. E. Weeks and D. J. Tannor, Chem. Phys. Lett., 1994, 224, 451

34 U. Hazi and H. S. Taylor, Phys. Rev. A, 1970, 1, 1109: W. P. Reinhardt, Annu. Rev. Phys. Chem., 1982, 33, 223; Y. K. Ho, Phys. Rep., 1983, 99, 1.

35 R. Sadeghi and R. T. Skodje, J. Chem. Phys., 1993, 99, 5126.

36 R. Sadeghi and R. T. Skodje, J. Chem. Phys., 1995, 102, 193.

37 A. Isele, C. Meier, V. Engel, N. Fahrer and Ch. Schlier, J. Chem. Phys., 1994, 101, 5919.

38 J. Dai and J. Z. H. Zhang, J. Chem. Phys., 1996, 104, 3664.

39 M. D. Feit, J. A. Fleck and A. Steiger, J. Comput. Phys. 1982, 47, 412.

40 P. Gaspard and S. A. Rice, J. Chem. Phys., 1989, 90, 2242.

41 I. Burghardt and P. Gaspard, J. Chem. Phys., 1994, 100, 6395.

42 M. F. Herman, J. Chem. Phys., 1986, 85, 2069.

43 F. T. Wall and R. N. Porter, J. Chem. Phys., 1962, 36, 3256.

44 T. Seideman and W. H. Miller, J. Chem. Phys., 1992, 96, 4412.

45 B. H. Bransden and J. C. Joachain, Physics of Atoms and Molecules, Longman, New York, 1984, p. 671.

46 E. J. Heller, J. Chem. Phys. 1977, 67, 3339.

47 W. H. Press, B. P. Flannery, S. A. Teukolsky and W. T. Vetterling, Numerical Recipes: The Art of Scientific Computing, Cambridge University Press, Cambridge, 2nd edn., 1992.

48 M. Berblinger, Ch. Schlier and T. Weiss, Comput. Phys. Commun., 1997, 99.

49 M. Tuckerman, B. J. Berne and G. J. Martyna, J. Chem. Phys., 1992, 97, 1990.

50 N. T. Maitra and E. J. Heller, Classical, Semiclassical and Quantum Dynamics in Atoms, Lecture notes in Physics, ed. $\mathrm{H}$. Friedrich and B. Eckhardt, Springer-Verlag, Berlin, 1977.

51 B. J. Berne, in Multiple Time Scales, ed. J. U. Brackbill and B. I. Cohen, Academic, New York, 1985.

52 F. T. Smith, J. Chem. Phys., 1959, 31, 1352.

53 P. Pechukas, in Dynamics of Molecular Collisions, ed. W. H. Miller, Plenum, New York, 1976, part B, p. 269.

54 R. N. Porter and M. Karplus, J. Chem. Phys., 1964, 40, 1105.

55 M. L. Brewer, J. S. Hulme and D. Manolopoulos, J. Chem. Phys., submitted.

56 J. Römelt, Chem. Phys., 1983, 79, 197.

Paper 6/07595I; Received 7th November, 1996 